\title{
Low membranous expression of $\beta$-catenin and high mitotic count predict poor prognosis in endometrioid carcinoma of the ovary
}

\author{
Daniel G Rosen ${ }^{1}$, Zhihong Zhang ${ }^{2}$, Bin Chang ${ }^{3,5}$, Xuemei Wang ${ }^{4}, \mathrm{E} \mathrm{Lin}^{4}$ and Jinsong Liu*3 \\ ${ }^{1}$ Department of Pathology, Baylor College of Medicine, Houston, TX, USA; ${ }^{2}$ The First Affiliated Hospital of \\ Nanjing Medical University, Nanjing, People's Republic of China; ${ }^{3}$ Department of Pathology, The University \\ of Texas M. D. Anderson Cancer Center, Houston, TX, USA; ${ }^{4}$ Department of Biostatistics and Applied \\ Mathematics, The University of Texas M. D. Anderson Cancer Center, Houston, TX, USA and ${ }^{5}$ Department of \\ Pathology, Shihezi University School of Medicine, Shihezi, People's Republic of China
}

\begin{abstract}
Mutations in the $\beta$-catenin gene are common in ovarian endometrioid carcinoma. Few studies have addressed the association of $\beta$-catenin expression with tumor characteristics and patient outcome, yielding controversial results. The purpose of this study was to retrospectively assess the expression of $\beta$-catenin in ovarian endometrioid carcinoma and correlate its expression with the Gynecologic Oncology Group's (GOG) grading system, clinicopathological characteristics, and patient survival. A total of 49 patients with primary ovarian endometrioid carcinoma were included in this study. A four-tier score grading system was used for the membranous staining (negative, weak, moderate, and strong) and the percentage of positive cells for the nuclear staining of $\beta$-catenin. The status of five morphometric parameters, nuclear morphology (uniform or pleomorphic), mitotic count, glandular pattern, degree of squamous differentiation, and status of papillary pattern, was assessed. We found that a low membranous expression of $\beta$-catenin and a high mitotic count ( $>15$ per 10 high-power fields) were significantly associated with poor prognosis and early recurrence of ovarian endometrioid carcinoma. In addition, cases with nuclear expression of $\beta$-catenin showed an intermediate overall survival risk and late disease recurrence. Young age at the time of diagnosis, advanced disease stage, and suboptimal debulking were among the clinical factors predicting poor survival and early disease recurrence. The presence of squamous differentiation, a papillary pattern or nuclear pleomorphism did not show any correlation with overall survival or disease-free survival. Low membranous expression of $\beta$-catenin and high mitotic count are poor prognostic indicators in patients with ovarian endometrioid carcinoma, whereas the GOG grading system showed no prognostic value. Our data suggest that there is a need to define a better grading system for ovarian endometrioid carcinoma. Molecular markers such as $\beta$-catenin and mitotic count could aid in defining this grading system.
\end{abstract}

Modern Pathology (2010) 23, 113-122; doi:10.1038/modpathol.2009.141; published online 9 October 2009

Keywords: ovarian endometrioid carcinoma; $\beta$-catenin; tumor grade; prognostic factors

Ovarian carcinomas constitute a heterogeneous group of neoplasms with different morphological characteristics, biological behaviors, and clinical outcomes. Endometrioid carcinomas account for $20-25 \%$ of ovarian carcinoma, surpassed in fre-

${ }^{*}$ Correspondence: Dr J Liu, MD, PhD, Department of Pathology, Unit 085, The University of Texas M. D. Anderson Cancer Center, 1515 Holcombe Blvd., Houston, TX 77030, USA. E-mail: jliu@mdanderson.org

Received 16 January 2009; revised 5 August 2009; accepted 6 August 2009; published online 9 October 2009 quency only by the serous histotype. ${ }^{1}$ Ovarian endometrioid carcinomas are most common in women in their 50s (mean age at diagnosis, 56 years). Endometriosis, which may be extraovarian, may occur in the ipsilateral or contralateral ovary, or may occur within the tumor itself, is found in $15-20 \%$ of the patients with ovarian endometrioid carcinoma. Approximately $14 \%$ of the cases also have synchronous endometrial carcinoma of the uterus. Ovarian endometrioid carcinoma has a 5 -year survival rate of $40-52 \%$ with most patients presenting with early stage disease. ${ }^{1,2}$ Some studies 
have showed that the endometrioid histology is associated with a better outcome than the serous histology. ${ }^{2-4}$ In contrast, other studies have shown that when patients with endometrioid and serous tumors of the ovary are matched for age, tumor grade, disease stage, and level of cytoreduction, there is no difference in either the rate of 5 -year survival or the mean/median length of survival. ${ }^{5}$

The most commonly used grading systems have been those of the International Federation of Gynecology and Obstetrics (FIGO), the World Health Organization (WHO), and the Gynecologic Oncology Group (GOG). ${ }^{6,7}$ The GOG grading system is the most widely used and is based on the FIGO grading system for endometrioid carcinomas of the endometrium. The GOG system is based on the tumor's architecture, with the overall grade modified by the nuclear grade when there is discordance; tumors that are architecturally grade 1 or 2 are increased by one grade in the presence of a notable degree of nuclear atypia. Well-differentiated endometrioid adenocarcinomas (GOG grade 1) account for most cases and are characterized by a confluent or cribriform proliferation of glands lined by a tall, stratified columnar epithelium with sharp luminal margins. Less than $5 \%$ of the tumors have a solid growth pattern. Mitotic figures are common. Squamous differentiation is present in $50 \%$ of the cases. In moderately differentiated carcinomas (grade 2) $6-50 \%$ of the tumors have a solid growth pattern. Poorly differentiated carcinomas (grade 3) show a solid and complex glandular growth pattern. Nuclear pleomorphism and mitotic activity are marked, and necrosis and hemorrhage are often prominent. ${ }^{8}$ Despite its widespread use, this grading system can be difficult to apply because of several pitfalls. One of the most controversial issues is its inter-observer reproducibility, which has reached unacceptable levels in some studies. , $^{6,10}$

Recent advances in molecular techniques have shed some light on the molecular events that lead to ovarian carcinoma development. However, no molecular marker is generally accepted as a prognostic indicator. $\beta$-catenin gene mutations are common molecular alterations in endometrioid carcinomas and therefore have emerged as a promising prognostic marker of this tumor. ${ }^{11-15} \beta$-Catenin has been implicated in two important biological processes: cell-cell adhesion and signal transduction. ${ }^{16}$ In adherens junctions of epithelial cells, $\beta$-catenin association with the cytoplasmic domain of cadherins has an important function in $\mathrm{Ca}^{2+}$-dependent cell adhesion. In the nucleus, $\beta$-catenin participates in the transduction of signals binding to the DNAactivating transcription. Deregulation of the cadherin/catenin complex has been implicated in cancer development, progression, differentiation, invasion, and metastatic potential in several malignancies. ${ }^{17-20}$ Deregulation of $\beta$-catenin has been described to be caused by oncogenic mutation of the $\beta$-catenin gene
(CTNNB1), mutations in the $A P C$ gene, or alterations of the Wnt signal transduction pathway. ${ }^{21,22}$

Recent studies have estimated that the mutation frequency of $\beta$-catenin in ovarian endometrioid carcinoma ranges from 16 to $54 \%{ }^{11,18,23,24}$ However, the prognostic significance of the expression of $\beta$-catenin is still a matter of controversy. The purpose of this study was to assess the expression of $\beta$-catenin in endometrioid ovarian carcinoma, correlate its expression with GOG grading system, clinicopathological characteristics, and patient survival.

\section{Materials and methods}

\section{Patient Selection}

Patients with primary endometrioid carcinoma of the ovary who underwent initial surgery at The University of Texas M. D. Anderson Cancer Center from 1990 to 2004 were retrospectively selected for this study by review of electronic charts. Only cases of pure endometrioid carcinoma were included in the final analysis, whereas those presenting as mixed-type carcinomas with an endometrioid component were excluded. Patient charts were reviewed to exclude those cases that clinically could have represented other primary sites, such as the colon. Immunohistochemical stains had been performed if necessary at diagnosis to exclude other primary sites. A total of 49 patients were identified for this study, for which hematoxylin- and eosin-stained and paraffin blocks were available. Follow-up information was updated through January 2006 by reviewing the patients' medical records. Demographic and survival data were entered into a comprehensive database created with Microsoft Access (version 97; Microsoft Corp., Redmond, WA, USA). Histopathologic diagnoses were based on WHO criteria; ${ }^{25}$ tumor grades were based on GOG criteria (see introduction); and disease stages were assessed according to the FIGO system. ${ }^{26-30}$ Diseasespecific survival rates were calculated as the percentage of subjects who survived with the disease for a defined period (reported as time since diagnosis), and only deaths from the disease were counted. Time to progression (disease-free survival) was defined as the interval from the date of treatment initiation until the disease progression was clinically evidenced by a $25 \%$ or greater increase in tumor size, appearance of new lesions, or a rise of serum CA 125 level to more than twice the upper limit of normal. ${ }^{31,32}$ Mean time to progression was calculated as the average time from diagnosis until the date of relapse. To analyze response to primary therapy, we grouped patients as responders or non-responders to the primary therapy. The latter group was also subdivided into groups of patients with progressive and relapsed disease. Patients in the progressive disease group were identified as those in whom no remission of 
the disease was observed after treatment initiation. Patients in the relapsed disease group were described as those in whom a remission of the disease was clinically documented. The extent of cytoreduction was defined as optimal if the largest diameter of any residual lesion from surgery was smaller than $1 \mathrm{~cm}$ or suboptimal if residual disease was larger than $1 \mathrm{~cm} .{ }^{33,34}$ The use of tissue block and chart review was approved by the Institutional Review Board at M. D. Anderson Cancer Center.

\section{Construction of Tissue Microarrays}

Tissue blocks were stored under ambient conditions at approximately $24^{\circ} \mathrm{C}$. A pathologist reviewed hematoxylin- and eosin-stained sections to select representative areas of tumor from which cores could be acquired for microarray analysis. Tissue microarray blocks were constructed as described earlier $^{35}$ with a precision instrument (Beecher Instruments, Silver Spring, MD, USA) that uses two separate core needles for punching the donor and recipient blocks and a micrometer-precise coordinate system for assembling tissue samples on a block. For each case, two replicate $1 \mathrm{~mm}$ corediameter samples were collected, and each was placed on a separate recipient block. The final tissue microarray consisted of one block containing duplicates of 49 spots. All samples were spaced $0.5 \mathrm{~mm}$ apart. Five-micrometer sections were obtained from the microarray and stained with hematoxylin and eosin to confirm the presence of tumors and to assess the tumors' histology. Tumor samples were randomly arranged on the blocks.

Sample tracking was based on coordinate positions for each tissue spot in the tissue microarray block; the spots were transferred onto tissue microarray slides for staining. This sample tracking system was linked to a Microsoft Access database containing demographic, clinicopathologic, and survival data on the subjects who provided the samples, thereby allowing rapid links between histologic data and clinical features. The array was read according to the given tissue microarray map; each core was scored and the results are presented as the mean of the two replicate core samples. Cases in which no tumor was found or no cores were available were excluded from the final data analysis.

\section{Immunohistochemical Analysis}

The tissue microarray slides were subjected to immunohistochemical staining as follows. After initial deparaffinization, endogenous peroxidase activity was blocked with $0.3 \%$ hydrogen peroxide. Deparaffinized sections were microwaved in $10 \mathrm{mM}$ citrate buffer ( $\mathrm{pH} \mathrm{6.0)}$ to unmask the epitopes. The slides were then incubated for $1 \mathrm{~h}$ at room temperature with monoclonal antibody against $\beta$-catenin (BD Biosciences Pharmingen, San Diego, CA, USA), then with biotin-labeled secondary antibody for $20 \mathrm{~min}$, and finally with a 1:40 solution of streptavidin:peroxidase for $20 \mathrm{~min}$. Tissues were then stained for 5 min with $0.05 \% 3^{\prime}, 3$-diaminobenzidine tetrahydrochloride that had been freshly prepared in $0.05 \mathrm{M}$ Tris buffer at $\mathrm{pH} 7.6$ containing $0.024 \% \mathrm{H}_{2} \mathrm{O}_{2}$ and then were counterstained with hematoxylin, dehydrated, and mounted. All the dilutions of antibody, biotin-labeled secondary antibody, and streptavidin-peroxidase were made in phosphatebuffered saline (pH 7.4) containing 1\% bovine serum albumin.

\section{Score Scoring System}

Four trained pathologists independently reviewed the slides for all 49 cases (DGR, ZZ, BC, and JL). An average of 16 slides (range, 1-80) were reviewed for each case and graded using GOG criteria. $^{7}$ In addition, the status of five morphometric parameters-including nuclear morphology (uniform or pleomorphic), mitotic count (per 10 high-power fields), glandular pattern ( $>50$ or $\leq 50 \%$ of glands), extent of squamous differentiation ( $\geq 20$ or $\leq 20 \%$ ), and status of papillary pattern (absent or present)and $\beta$-catenin expression were visually analyzed and recorded separately. Membranous and nuclear expressions were analyzed separately in individual experiments. Samples in which more than $10 \%$ of nuclei stained were judged to be positive and labeled as 'positive' regardless of the membranous staining. The remaining cases in which no more than $10 \%$ of nuclei were stained or no nuclear staining was observed were labeled as 'negative.' Cytoplasmic membranous staining was scored as follows: no staining or membrane staining in less than $10 \%$ of the tumor cells was considered negative (0); weak noticeable membranous staining in more than $10 \%$ of the tumor cells was defined as $1+$; weak to moderate complete membrane staining in more than $10 \%$ of the tumor cells was defined as $2+$; and strong complete membrane staining in more than $10 \%$ of the tumor cells was defined as $3+$ (Figure 1).

Immunohistochemical staining was performed in whole sections in nine cases in which both cores showed weak membranous staining and no nuclear staining. In all nine cases, the whole sections confirmed the findings of those seen in the tissue microarray cores.

\section{Statistical Analysis}

Descriptive statistics were calculated; Wilcoxon signed-rank sum tests were performed to assess the association between continuous variables in subgroups; $\chi^{2}$ tests or Fisher's exact tests were used to assess the association between categorical variables; $\kappa$ statistics were used to assess the agreement between two categorical variables; McNemar's tests 

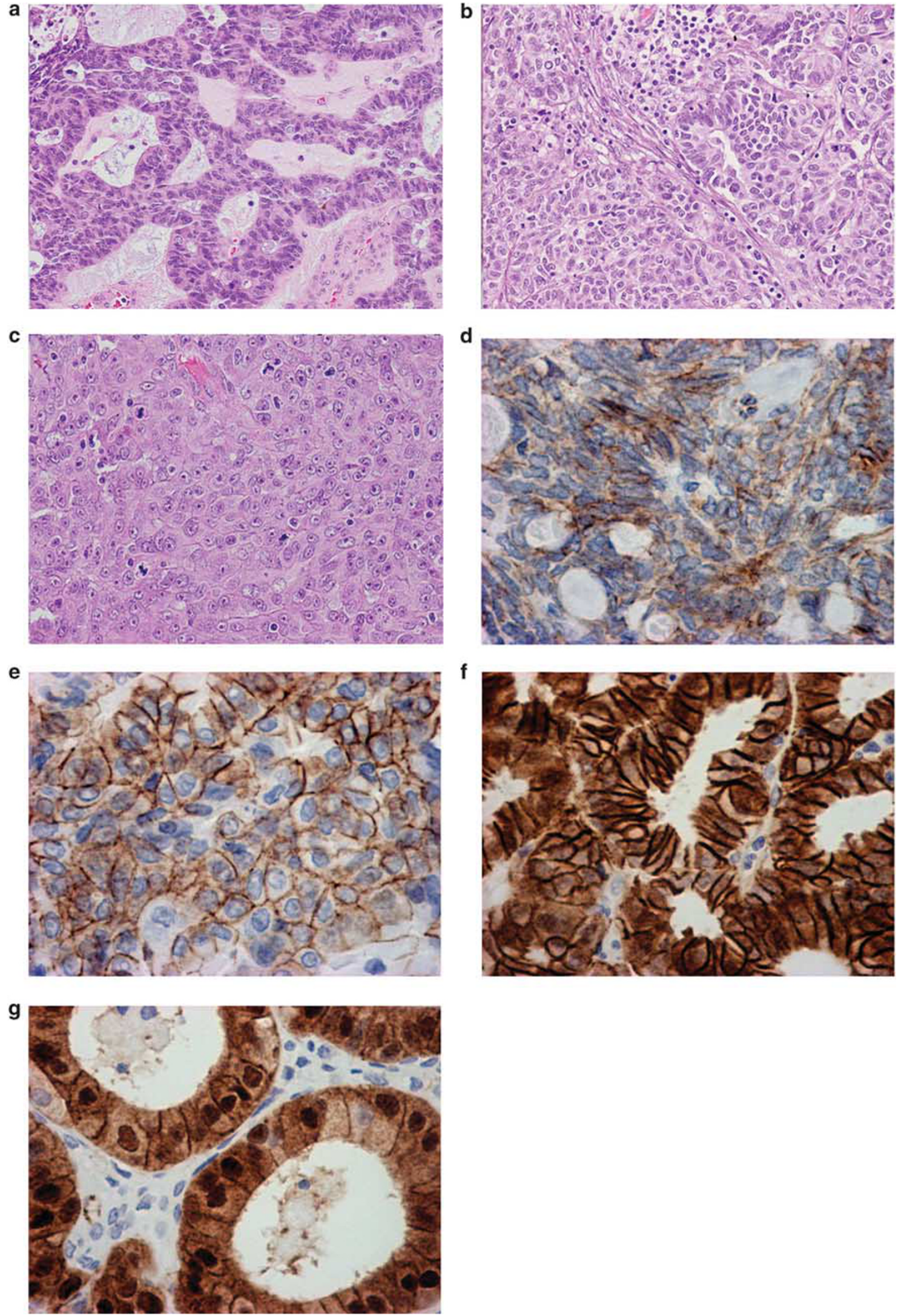

Figure 1 Photomicrographs examples of (a) GOG grade 1 (H\&E, $\times 40)$, (b) GOG grade $2(\mathrm{H} \& \mathrm{E}, \times 40)$, and $(\mathbf{c})$ GOG grade $3(\mathrm{H} \& \mathrm{E}, \times 40)$ endometrioid carcinoma of the ovary. Examples of immunostaining against $\beta$-catenin showing $(\mathbf{d})$ weak membranous staining $(\times 40)$, $(\mathbf{e})$ moderate membranous staining $(\times 40)$, (f) strong membranous staining $(\times 40)$, and $(\mathrm{g})$ nuclear staining $(\times 40)$. 
were used to assess the homogeneity between paired categorical variables; linear regression methods were used to assess the agreement of two continuous variables by testing intercepts equal to 0 and coefficients equal to 1; Kaplan-Meier methods were used to evaluate survival; and log-rank tests were used to compare the differences in survival between groups. In addition, univariate and multivariate Cox proportional hazards regression modeling was used to estimate the hazard ratios of death and relapse, including 95\% confidence intervals. All computations were carried out using SAS version 8.0 (Cary, NC, USA) or S-PLUS 8 (Palo Alto, CA, USA).

\section{Results}

Correlation Between FIGO Stage and the Phenotypic Expression of $\beta$-Catenin, Patient Characteristics, and Tumor Morphology

The phenotypic expression of $\beta$-catenin in tumor cells was quite variable (Table 1). Examples of the immunostaining patterns are shown in Figure 1. Membrane expression was present in all 49 ovarian endometrioid carcinoma cases, most of which had moderate to strong staining (39 cases, 79.9\%). A total of 19 cases (38.7\%) also co-expressed $\beta$-catenin nuclear staining, ranging from 1 to $80 \%$ of positive nuclei. Most of the cases showing nuclear staining also had moderate to strong membranous staining. Only one case showed weak membranous staining and nuclear positivity. The phenotypic expression of $\beta$-catenin was not associated with any of the tested variables presented on Table 1. However, there was a trend for higher membranous and nuclear expression in grade 3 tumors than in grades 1 and 2 tumors (Table 1 ).

\section{Association Between Covariates and Survival}

The univariate and multivariate models used to estimate the association between covariates and overall survival are shown in Figure 2 and Table 2. GOG grade was not correlated with patient survival (Figure 2a). In contrast, patients with low membranous expression of $\beta$-catenin showed poor survival compared with those who had strong membranous and nuclear expression $(P=0.022)$ (Figure 2b). Further analysis showed that patients with stage III or IV disease had an approximately 5.3 times higher risk of death than patients with stage I or II carcinoma. The levels of cytoreduction, mitotic count, and membranous expression of $\beta$-catenin were also significantly associated with survival. Patients with suboptimal surgery had an approximately 10.6 times higher risk of death than patients with optimal surgery. Patients with a mitotic count greater than 15 had approximately 3.3 times higher risk of death than patients with a mitotic count less than or equal to 15 . Patients with low $\beta$-catenin membranous expression had approximately 7.1 times higher risk of death than patients with moderate to high membranous expression. None of the other tested variables reached statistical significance in the univariate analysis. In the multivariate analysis of all factors affecting overall survival, only late disease stage, suboptimal debulking surgery, high mitotic count, and low membranous expression of $\beta$-catenin remained statistically significant and were associated with poor outcome.

\section{Association Between Covariates and Disease-Free Survival}

Figure 2 and Table 3 show correlations between covariates and disease-free survival. No correlation was found in the univariate analysis between the GOG grade and disease-free survival (Figure 2b). In contrast, patients with strong membranous expression of $\beta$-catenin lived longer without disease than patients with low membranous expression or nuclear expression $(P=0.035)$ (Figure $2 \mathrm{~d})$. Further analysis showed that patients who had tumor recurrence were significantly younger than patients without recurrence $(P=0.021)$; with a 1 -year increase in age, the recurrence rate decreased approximately $5 \%$. Patients with stage III or IV had an approximately 3.6 times higher risk of recurrence and a significantly higher percentage of recurrence than patients with stage I or II $(P=0.018)$. Patients with suboptimal surgery had a 6.3 times higher risk of recurrence and a significantly higher percentage of recurrence than patients with optimal surgery $(P=0.001)$. Patients with mitotic count greater than 15 per HPF had an approximately 2.7 times higher risk of recurrence and had a significantly higher percentage of recurrence than patients with a mitotic count less than or equal to 15 per HPF $(P=0.017)$. Patients with recurrence had a significantly lower nuclear expression of $\beta$-catenin than patients without recurrence $(P=0.009)$ (Table 3$)$. In the multivariate analysis of all factors affecting disease-free survival, only age at presentation, disease stage, and debulking surgery remained statistically significant.

\section{Discussion}

In this study, we analyzed the immunohistochemical expression of $\beta$-catenin in 49 cases of ovarian endometrioid carcinoma, assessed its association with clinicopathological variables including the GOG grading system, and evaluated its impact on overall survival and disease-free survival. We found that of all the morphologic factors analyzed, only low membranous expression of $\beta$-catenin and high mitotic count were significantly associated with poor prognosis in ovarian endometrioid carcinoma. The presence of squamous differentiation, a highly glandular pattern, papillary pattern, or nuclear 
Table 1 Correlation between $\beta$-catenin expression and clinicopathological characteristics

\begin{tabular}{|c|c|c|c|c|c|c|c|c|c|}
\hline & \multicolumn{3}{|c|}{ Membrane expression } & \multicolumn{6}{|c|}{ Nuclear expression } \\
\hline & 1 & 2 & 3 & 0 & $1-10 \%$ & $11-20 \%$ & $21-50 \%$ & $\geqslant 51 \%$ & Total \\
\hline \multicolumn{10}{|l|}{ GOG grade } \\
\hline 1 & $2(25 \%)$ & $1(12 \%)$ & $5(63 \%)$ & $5(64 \%)$ & $1(12 \%)$ & $1(12 \%)$ & $1(12 \%)$ & 0 & 8 \\
\hline 2 & $7(30 \%)$ & $4(17 \%)$ & $12(53 \%)$ & $14(71 \%)$ & 0 & $6(26 \%)$ & $3(13 \%)$ & 0 & 23 \\
\hline 3 & $1(5 \%)$ & $3(17 \%)$ & $14(78 \%)$ & $11(61 \%)$ & 0 & $1(6 \%)$ & $2(11 \%)$ & $4(22 \%)$ & 18 \\
\hline$P$-value & & 0.32 & & & & 0.32 & & & \\
\hline \multicolumn{10}{|l|}{ FIGO stage } \\
\hline I & $1(7 \%)$ & $3(20 \%)$ & $11(73 \%)$ & $11(73 \%)$ & $1(7 \%)$ & $3(20 \%)$ & 0 & 0 & 15 \\
\hline II & $3(23 \%)$ & $2(15 \%)$ & $8(62 \%)$ & $6(46 \%)$ & 0 & $4(31 \%)$ & $3(23 \%)$ & 0 & 13 \\
\hline III & $3(23 \%)$ & $3(23 \%)$ & $7(54 \%)$ & $8(62 \%)$ & 0 & 0 & $2(15 \%)$ & $3(23 \%)$ & 13 \\
\hline IV & $3(37 \%)$ & 0 & $5(63 \%)$ & $5(62 \%)$ & 0 & $1(12 \%)$ & $1(12 \%)$ & $1(12 \%)$ & 8 \\
\hline$P$-value & & 0.56 & & & & 0.17 & & & \\
\hline \multicolumn{10}{|l|}{ FIGO stage } \\
\hline Early & $4(14 \%)$ & $5(18 \%)$ & $19(68 \%)$ & $17(61 \%)$ & $1(3 \%)$ & $7(25 \%)$ & $3(11 \%)$ & 0 & 28 \\
\hline Late & $6(29 \%)$ & $3(14 \%)$ & $12(57 \%)$ & $13(62 \%)$ & 0 & $1(5 \%)$ & $3(14 \%)$ & $4(19 \%)$ & 21 \\
\hline$P$-value & & 0.46 & & & & 0.06 & & & \\
\hline \multicolumn{10}{|c|}{ Patient's age at diagnosis } \\
\hline$<58$ & $4(16 \%)$ & $5(20 \%)$ & $16(64 \%)$ & $18(72 \%)$ & 0 & $3(12 \%)$ & $2(8 \%)$ & $2(8 \%)$ & 25 \\
\hline$>59$ & $6(25 \%)$ & $3(12 \%)$ & $15(63 \%)$ & $12(50 \%)$ & $1(4 \%)$ & $5(21 \%)$ & $4(17 \%)$ & $2(8 \%)$ & 24 \\
\hline$P$-value & & 0.66 & & & & 0.5 & & & \\
\hline \multicolumn{10}{|l|}{ Relapse } \\
\hline No & $3(12 \%)$ & $5(20 \%)$ & $17(68 \%)$ & $13(52 \%)$ & $1(4 \%)$ & $5(20 \%)$ & $4(16 \%)$ & $2(8 \%)$ & 25 \\
\hline Yes & $3(23 \%)$ & $3(23 \%)$ & $7(54 \%)$ & $10(76 \%)$ & 0 & $1(8 \%)$ & $1(8 \%)$ & $1(8 \%)$ & 13 \\
\hline Progressive disease & $2(40 \%)$ & 0 & $3(60 \%)$ & $5(100 \%)$ & 0 & 0 & 0 & 0 & 5 \\
\hline$P$-value & & 0.49 & & & & 0.66 & & & \\
\hline \multicolumn{10}{|l|}{ Type of surgery } \\
\hline Optimal & $4(12 \%)$ & $6(17 \%)$ & $24(71 \%)$ & $20(59 \%)$ & $1(3 \%)$ & $6(17 \%)$ & $4(12 \%)$ & $3(9 \%)$ & 34 \\
\hline Suboptimal & $4(36 \%)$ & $2(18 \%)$ & $5(46 \%)$ & $8(73 \%)$ & 0 & $2(18 \%)$ & $1(9 \%)$ & 0 & 11 \\
\hline$P$-value & & 0.15 & & & & 0.8 & & & \\
\hline \multicolumn{10}{|l|}{ Squamous component } \\
\hline No & $9(26 \%)$ & $6(8 \%)$ & 19 (56\%) & $23(67 \%)$ & 0 & $5(15 \%)$ & $3(9 \%)$ & $3(9 \%)$ & 34 \\
\hline Yes & $1(7 \%)$ & $2(13 \%)$ & $12(80 \%)$ & $7(46 \%)$ & $1(7 \%)$ & $3(20 \%)$ & $3(20 \%)$ & $1(7 \%)$ & 15 \\
\hline$P$-value & & 0.24 & & & & 0.36 & & & \\
\hline \multicolumn{10}{|l|}{ Papillary component } \\
\hline No & $4(20 \%)$ & $2(10 \%)$ & $14(70 \%)$ & $12(60 \%)$ & 0 & $4(20 \%)$ & $2(10 \%)$ & $2(10 \%)$ & 20 \\
\hline Yes & $6(21 \%)$ & $6(21 \%)$ & $17(58 \%)$ & $18(62 \%)$ & $1(3 \%)$ & $4(14 \%)$ & $4(14 \%)$ & $2(7 \%)$ & 29 \\
\hline$P$-value & & 0.65 & & & & 0.86 & & & \\
\hline \multicolumn{10}{|l|}{ Nuclear pleomorphism } \\
\hline No & $4(15 \%)$ & $4(15 \%)$ & 19 (70\%) & $14(52 \%)$ & $1(4 \%)$ & $6(22 \%)$ & $4(15 \%)$ & $2(7 \%)$ & 27 \\
\hline Yes & $6(27 \%)$ & $4(18 \%)$ & $12(55 \%)$ & $16(73 \%)$ & 0 & $2(9 \%)$ & $2(9 \%)$ & $2(9 \%)$ & 22 \\
\hline$P$-value & & 0.51 & & & & 0.5 & & & \\
\hline \multicolumn{10}{|l|}{ Glandular component } \\
\hline$<50 \%$ & $4(15 \%)$ & $5(19 \%)$ & $17(66 \%)$ & $18(69 \%)$ & 0 & $3(11.5 \%)$ & $2(8 \%)$ & $3(11.5 \%)$ & 26 \\
\hline$>51 \%$ & $6(26 \%)$ & $3(13 \%)$ & $14(61 \%)$ & $12(52 \%)$ & $1(4 \%)$ & $5(22 \%)$ & $4(18 \%)$ & $1(4 \%)$ & 23 \\
\hline$P$-value & & 0.71 & & & & 0.37 & & & \\
\hline \multicolumn{10}{|l|}{ Mitosis (10HPF) } \\
\hline $1-5$ & $7(28 \%)$ & $3(12 \%)$ & $15(60 \%)$ & $14(56 \%)$ & $1(4 \%)$ & $5(20 \%)$ & $3(12 \%)$ & $2(8 \%)$ & 25 \\
\hline $6-10$ & $1(14 \%)$ & $3(43 \%)$ & $3(43 \%)$ & $4(57 \%)$ & 0 & $2(29 \%)$ & $1(14 \%)$ & 0 & 7 \\
\hline $10-20$ & $1(20 \%)$ & $2(40 \%)$ & $2(40 \%)$ & $4(80 \%)$ & 0 & 0 & 0 & $1(20 \%)$ & 5 \\
\hline$>21$ & $1(8 \%)$ & 0 & $11(92 \%)$ & $8(67 \%)$ & 0 & $1(8 \%)$ & $2(17 \%)$ & $1(8 \%)$ & 12 \\
\hline$P$-value & & 0.46 & & & & 0.92 & & & \\
\hline Total & $10(21 \%)$ & $8(16 \%)$ & $31(63 \%)$ & $30(61 \%)$ & $1(2 \%)$ & $8(17 \%)$ & $6(12 \%)$ & $4(8 \%)$ & 49 \\
\hline
\end{tabular}

pleomorphism was associated with neither membranous nor nuclear expression of $\beta$-catenin. Young age at diagnosis, advanced disease stage, and suboptimal debulking surgery were also among the clinical factors predicting poor survival and early disease recurrence. 

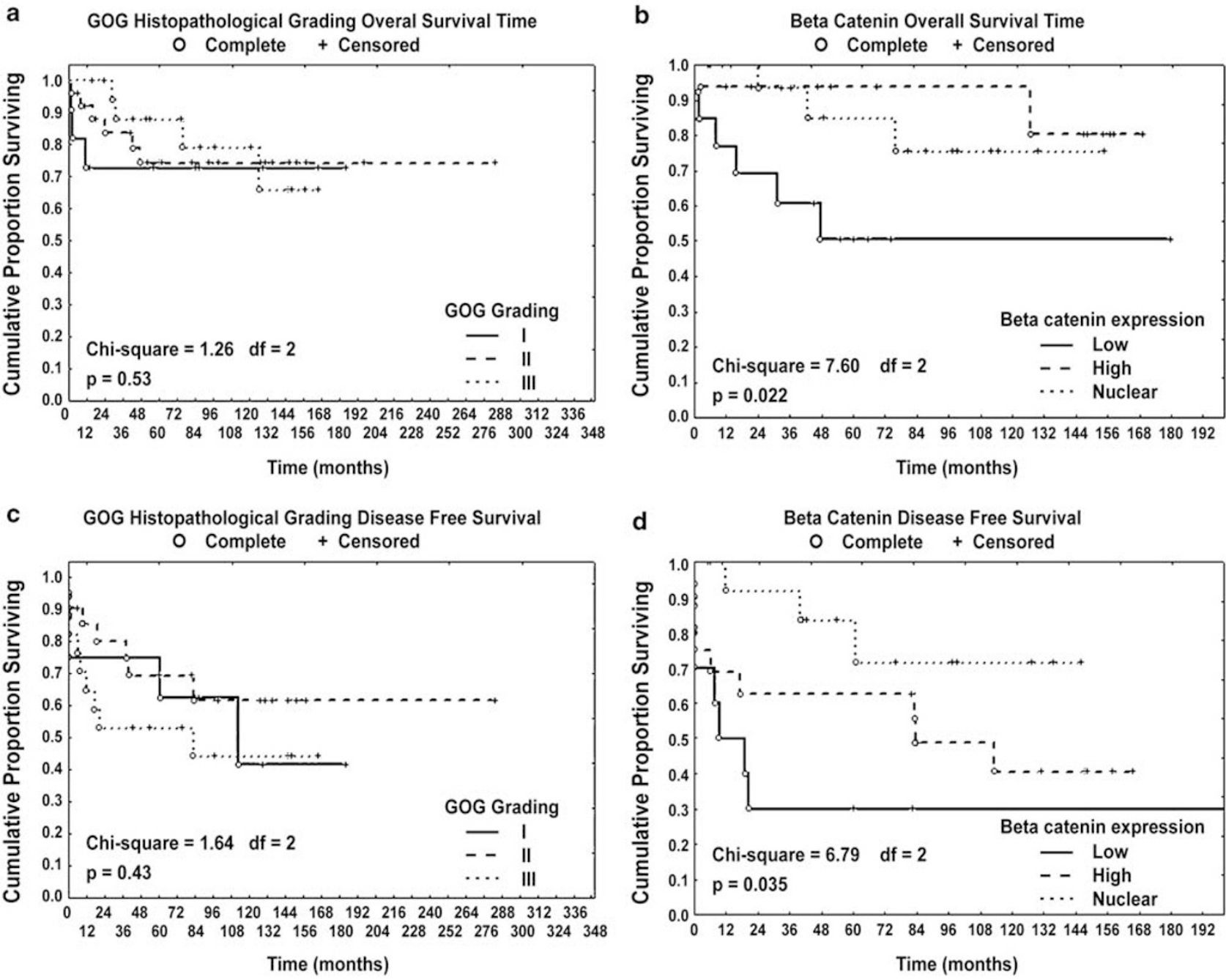

Figure 2 Kaplan-Meier estimates for (a) GOG grading system overall survival, (b) $\beta$-catenin overall survival, (c) GOG grading system disease-free survival, and (d) $\beta$-catenin disease-free survival.

Table 2 Univariate and multivariate Cox proportional hazards models to estimate the association between covariates and overall survival

\begin{tabular}{|c|c|c|c|c|c|c|c|c|}
\hline \multirow[t]{2}{*}{ Variables } & \multicolumn{4}{|c|}{ Univariate } & \multicolumn{4}{|c|}{ Multivariate } \\
\hline & Estimate & s.e. & $\mathrm{P}$ & $H R(95 \% C I)$ & Estimate & s.e. & $\mathrm{P}$ & $H R(95 \% C I)$ \\
\hline Age (1-year increase) & 0.02 & 0.02 & 0.39 & $1.0(0.9-1.0)$ & 0.15 & 0.05 & 0.006 & $1.1(1.0-1.3)$ \\
\hline Stage $(3,4$ vs 1,2$)$ & 1.68 & 0.60 & 0.005 & $5.3(1.6-17.5)$ & 2.25 & 1.02 & 0.027 & $9.4(1.2-70.1)$ \\
\hline Surgery (suboptimal vs optimal) & 2.36 & 0.63 & 0.0002 & $10.6(3.0-36.7)$ & 1.99 & 0.92 & 0.031 & $7.3(1.1-44.9)$ \\
\hline Squamous (yes vs no) & -0.50 & 0.65 & 0.44 & $0.6(0.1-2.1)$ & - & - & - & - \\
\hline Papillary (yes vs no) & -0.25 & 0.55 & 0.64 & $0.7(0.2-2.3)$ & - & - & - & - \\
\hline Pleom (yes vs no) & 0.54 & 0.57 & 0.33 & $1.7(0.5-5.2)$ & - & - & - & - \\
\hline Glands $(>50$ vs $<=50)$ & -0.60 & 0.57 & 0.28 & $0.5(0.1-1.6)$ & - & - & - & - \\
\hline Mitosis $(>15$ vs $<=15)$ & 1.21 & 0.56 & 0.031 & $3.3(1.1-10.1)$ & 2.19 & 0.93 & 0.019 & $8.9(1.4-56.0)$ \\
\hline$\beta$-catenin & 1.9 & 0.60 & 0.001 & $7.1(2.17-23.8)$ & 2.66 & 0.90 & 0.003 & $14.2(2.4-82.3)$ \\
\hline
\end{tabular}

Although tumor grade predicts prognostic significance in several cancers, such as breast, prostate, and endometrium, the importance of tumor grade in ovarian endometrioid carcinoma remains a controversial topic. Several grading systems have been proposed by many groups, including a universal grading system irrespective of the histologic type. ${ }^{36}$
In almost all the reported studies, grade showed at least some correlation with prognosis. ${ }^{6}$ However, it has been shown that only grading each histologic type as a unique entity will provide valuable information about the tumor's biological behavior. ${ }^{37}$ It is also generally accepted that each histologic subtype has a different biological behavior varying 
Table 3 Univariate and multivariate Cox proportional hazards models to estimate the association between covariates and disease free survival

\begin{tabular}{|c|c|c|c|c|c|c|c|c|}
\hline \multirow[t]{2}{*}{ Variables } & \multicolumn{4}{|c|}{ Univariate } & \multicolumn{4}{|c|}{ Multivariate } \\
\hline & Estimate & s.e. & $\mathrm{P}$ & $H R(95 \% C I)$ & Estimate & s.e. & $\mathrm{P}$ & $H R(95 \% C I)$ \\
\hline Age (1-year increase) & -0.05 & 0.02 & 0.021 & $0.94(0.9-0.9)$ & -0.08 & 0.04 & 0.024 & $0.92(0.8-0.9)$ \\
\hline Stage $(3,4$ vs 1,2$)$ & 1.29 & 0.55 & 0.018 & $3.62(1.2-10.5)$ & 0.94 & 0.63 & 0.138 & $2.56(0.7-8.8)$ \\
\hline Surgery (suboptimal vs optimal) & 1.85 & 0.56 & 0.001 & $6.34(2.1-19.0)$ & 1.39 & 0.65 & 0.032 & $4.01(1.1-14.3)$ \\
\hline Squamous (yes vs no) & -0.60 & 0.65 & 0.359 & $0.55(0.1-1.9)$ & - & - & - & - \\
\hline Papillary (yes vs no) & -0.28 & 0.54 & 0.607 & $0.75(0.2-2.1)$ & - & - & - & - \\
\hline Pleom (yes vs no) & -0.12 & 0.54 & 0.824 & $0.88(0.3-2.5)$ & - & - & - & - \\
\hline Glands $(>50$ vs $<=50)$ & -1.00 & 0.56 & 0.076 & $0.36(0.1-1.1)$ & - & - & - & - \\
\hline Mitosis $(>15$ vs $<=15)$ & 1.00 & 0.56 & 0.017 & $2.70(0.9-8.1)$ & - & - & - & - \\
\hline$\beta$-catenin & 0.98 & 0.66 & 0.009 & $2.67(0.7-9.8)$ & - & - & - & - \\
\hline
\end{tabular}

in aggressiveness, chemosensitivity, and metastatic potential. Even though a universal system is very desirable, current grading systems are still considered an unreliable factor on which to base a therapeutic decision. Not surprisingly, we did not find a correlation between the GOG grading system and patient survival.

Few studies have analyzed the prognostic significance of the actual grading system used for ovarian endometrioid carcinoma. In a study done by Kline et al including 145 cases of ovarian endometrioid carcinoma, no significant difference was found between grades 1 and 2, whereas this difference was significant for grade 3 tumors. ${ }^{38} \mathrm{In}$ another study by Grosso et al of 106 cases, tumor grade showed prognostic significance in the univariate analysis but not in the multivariate analysis of all factors affecting survival. ${ }^{39}$ In the study by Shimizu et $a{ }^{40}{ }^{40}$ nuclear grade and mitotic count were found to be independent from architectural features in ovarian endometrioid carcinoma. However, they were not found to be significant in the Cox multivariate analysis of survival time. Differences in results among studies probably reflect the different grading system criteria. When tested separately, mitotic count was more consistently found to be of prognostic relevance than nuclear pleomorphism and glandular pattern in most studies ${ }^{40-45} \mathrm{Fu}$ et $a l^{46}$ found a decreased 5-year survival rate in patients with squamous foci ( $21 \%$ 5-year survival rate) compared with patients with ovarian endometrioid adenoacanthoma (90\% 5-year survival rate). Other studies found that morphologic differences between atypical complex hyperplasia with and without squamous morules seemed to correlate with course of the disease. ${ }^{47}$ In our study, mitotic count was the only significant morphologic prognostic factor in both the univariate and multivariate analyses to predict the rate of disease-specific survival and disease-free survival in ovarian endometrioid carcinoma. The analysis of the remaining morphologic characteristics alone or in combination-namely presence of squamous component, glandular pattern, papillary pattern, and nuclear pleomorphism- did not show that they were associated with survival.

Previous studies by other investigators have shown that $\beta$-catenin has an important function in the wingless/Wnt signal transduction pathway. ${ }^{48}$ In the presence of Wnt, its receptor, Frizzled, in complex with LRP6, is activated. This activation leads to an activation of signaling cascade in which Dishevelled activates GBP, an inhibitor of GSK3 $\beta$. Consequently, $\beta$-catenin cannot be targeted for destruction by the proteosome and is free to diffuse into the nucleus, where it acts as a co-activator for TCF-responsive genes. ${ }^{48}$ If the Wnt signal is absent, then APC protein binds to $\beta$-catenin, promoting its degradation and inactivation. APC works with GSK3- $\beta$ to regulate $\beta$-catenin through potential phosphorylation of three serine and one threonine residues. $\beta$-Catenin gene mutation stabilizes $\beta$-catenin itself and causes it to accumulate within the cell and to translocate to nuclei through inhibition of its phosphorylation. Mutated $\beta$-catenin, in association with members of the Tcf-Lef family, functions as a transcriptional factor, providing transfer cell proliferation signals to the nucleus. ${ }^{48}$ Oncogenic mutations of the $\beta$-catenin gene have been detected in some types of cancers, such as endometrial endometrioid carcinoma, hepatocellular carcinoma, colorectal cancer, melanoma, and medulloblastoma. ${ }^{24,49-53}$ The mutation frequency of $\beta$-catenin in ovarian endometrioid carcinoma ranges from 16 to $54 \%,{ }^{11,18,23,24}$ suggesting that this gene has an important function in this subtype of ovarian cancer.

The prognostic significance of $\beta$-catenin expression and its association with patient outcome is still a matter of controversy. It has been previously reported that ovarian endometrioid carcinomas with exclusively membranous expression of $\beta$-catenin seem to represent a different subgroup of carcinomas that probably have a worse prognosis. ${ }^{18}$ This study's authors also stated that in early stage ovarian cancer, determination of the $\beta$-catenin expression pattern could prove to be useful in selecting low-risk patients,${ }^{18}$ although opposite reports have also been 
reported..$^{54-56}$ In our cohort, we found that patients exhibiting low membranous $\beta$-catenin expression had a poor outcome compared with those showing high membranous expression. In addition, patients showing nuclear staining were in an intermediate risk group. Furthermore, patients with low membranous expression relapsed earlier than patients with high membranous expression. Patients with nuclear expression lived longer without disease than patients in the other two groups. Our report provided a strong support that low membrane staining of $\beta$-catenin is associated with unfavorable prognostic significance in this subtype of ovarian cancer.

Despite the morphologic similarity between endometrioid carcinomas arising in the uterine cavity and those arising in the ovaries, there is a large body of evidence that these two entities differ at the molecular level. For instance, a higher percentage of $\beta$-catenin mutation is observed in synchronous tumors than in single ovarian carcinoma. ${ }^{57}$ In addition, ovarian cancers exhibit microsatellite instability and PTEN alterations less frequently than their uterine counterparts. ${ }^{13}$ The study of loss of heterozygosity in synchronous uterine and ovarian endometrioid tumors suggests that when is selective for one tumor site the patient is more likely to have two separate primary tumors. ${ }^{58}$ Owing to this molecular difference, the ovarian endometrioid carcinoma grading system might not be parallel to that for uterine endometrioid carcinomas. Therefore, using the same grading system for both entities might not be appropriate. As shown by our results, we could not show a correlation between patient survival and tumors grade determined using GOG system criteria. However, mitotic count and $\beta$-catenin expression did show independent prognostic value. Our results suggest that ovarian endometrioid carcinoma might have a unique biological behavior in which the proliferation status of the tumor is critical. Clearly, there is need to define a better grading system for ovarian endometrioid carcinoma. We believe that molecular markers such as $\beta$-catenin could aid in defining this grading system.

\section{Acknowledgement}

J.L. is supported by R01 and an Ovarian SPORE grant (IP50CA83638), and Cancer Center Core grant (CA016672) by from the National Cancer Institute.

\section{Disclosure/conflict of interest}

The authors declare no conflict of interest.

\section{References}

1 Blaustein A, Kurman RJ. Blaustein's Pathology of the Female Genital Tract 4th edn. Springer: New York, 2002.
2 Gilks CB, Ionescu DN, Kalloger SE, et al. Tumor cell type can be reproducibly diagnosed, is of independent prognostic significance in patients with maximally debulked ovarian carcinoma. Hum Pathol 2008; 39:1239-1251.

3 Storey DJ, Rush R, Stewart M, et al. Endometrioid epithelial ovarian cancer. Cancer 2008;112:2211-2220.

4 Winter 3rd WE, Maxwell GL, Tian C, et al. Prognostic factors for III stage epithelial ovarian cancer: a Gynecologic Oncology Group Study. J Clin Oncol 2007;25:3621-3627.

5 Zwart J, Geisler JP, Geisler HE. Five-year survival in patients with endometrioid carcinoma of the ovary versus those with serous carcinoma. Eur J Gynaecol Oncol 1998;19:225-228.

6 Silverberg SG. Histopathologic grading of ovarian carcinoma: a review, proposal. Int J Gynecol Pathol 2000;19:7-15.

7 Benda JA, Zaino R. GOG Pathology Manual. Gynecologic Oncology Group: Buffalo, NY, 1994.

8 Silverberg SG, Kurman RJ. Tumors of the Uterine Corpus, Gestational Trophoblastic Disease. Endometrial Carcinoma. In: Rosai J (ed). Armed Forces Institute of Pathology: Washington, DC, 1992, 54p.

9 Hernandez E, Bhagavan BS, Parmley $\mathrm{TH}$, et al. Interobserver variability in the interpretation of epithelial ovarian cancer. Gynecol Oncol 1984;17:117-123.

10 Hernandez E, Rosenshein NB, Bhagavan BS, et al. Tumor heterogeneity, histopathology in epithelial ovarian cancer. Obstet Gynecol 1984;63:330-334.

11 Saegusa M, Okayasu I. Frequent nuclear beta-catenin accumulation, associated mutations in endometrioidtype endometrial, ovarian carcinomas with squamous differentiation. J Pathol 2001;194:59-67.

12 Bell DA. Origins, molecular pathology of ovarian cancer. Mod Pathol 2005;18(Suppl 2):S19-S32.

13 Catasus L, Bussaglia E, Rodrguez I, et al. Molecular genetic alterations in endometrioid carcinomas of the ovary: similar frequency of beta-catenin abnormalities but lower rate of microsatellite instability, PTEN alterations than in uterine endometrioid carcinomas. Hum Pathol 2004;35:1360-1368.

14 Gatcliffe TA, Monk BJ, Planutis K, et al. Wnt signaling in ovarian tumorigenesis. Int J Gynecol Cancer 2008; 18:954-962.

15 Zhai Y, Wu R, Schwartz DR, et al. Role of beta-catenin/ T-cell factor-regulated genes in ovarian endometrioid adenocarcinomas. Am J Pathol 2002;160:1229-1238.

16 Peifer M. Cancer catenins, cuticle pattern: a complex connection. Science 1993;262:1667-1668.

17 Mirabelli-Primdahl L, Gryfe R, Kim H, et al. Betacatenin mutations are specific for colorectal carcinomas with microsatellite instability but occur in endometrial carcinomas irrespective of mutator pathway. Cancer Res 1999;59:3346-3351.

18 Gamallo C, Palacios J, Moreno G, et al. beta-catenin expression pattern in stage I II ovarian carcinomas : relationship with beta-catenin gene mutations, clinicopathological features,, clinical outcome. Am J Pathol 1999;155:527-536.

19 Nhieu JT, Renard CA, Wei Y, et al. Nuclear accumulation of mutated beta-catenin in hepatocellular carcinoma is associated with increased cell proliferation. Am J Pathol 1999;155:703-710.

20 Kobayashi K, Sagae S, Nishioka Y, et al. Mutations of the beta-catenin gene in endometrial carcinomas. Jpn J Cancer Res 1999;90:55-59. 
$21 \mathrm{Wu}$ R, Zhai Y, Fearon ER, et al. Diverse mechanisms of beta-catenin deregulation in ovarian endometrioid adenocarcinomas. Cancer Res 2001;61:8247-8255.

22 Morin PJ, Sparks AB, Korinek V, et al. Activation of betacatenin-Tcf signaling in colon cancer by mutations in beta-catenin or APC. Science 1997;275:1787-1790.

23 Sagae S, Kobayashi K, Nishioka Y, et al. Mutational analysis of beta-catenin gene in Japanese ovarian carcinomas: frequent mutations in endometrioid carcinomas. Jpn J Cancer Res 1999;90:510-515.

24 Palacios J, Gamallo C. Mutations in the beta-catenin gene (CTNNB1) in endometrioid ovarian carcinomas. Cancer Res 1998;58:1344-1347.

25 Tavassoli FA, Devilee P, World Health O, International Agency for Research on C. Pathology and Genetics of Tumours of the Breast and Female Genital Organs. Lyon: IAPS Press, 2003.

26 Scully RE. World Health Organization classification and nomenclature of ovarian cancer. Natl Cancer Inst Monogr 1975;42:5-7.

27 Russell P. The pathological assessment of ovarian neoplasms. II: the proliferating 'epithelial' tumours. Pathology 1979;11:251-282.

28 Russell P. The pathological assessment of ovarian neoplasms. III: the malignant 'epithelial' tumours. Pathology 1979;11:493-532.

29 Russell P. The pathological assessment of ovarian neoplasms. I: introduction to the common 'epithelial' tumours and analysis of benign 'epithelial' tumours. Pathology 1979;11:5-26.

30 Shepherd JH. Revised FIGO staging for gynaecological cancer. Br J Obstet Gynaecol 1989;96:889-892.

31 Armstrong DK. Relapsed ovarian cancer: challenges and management strategies for a chronic disease. Oncologist 2002;7(Suppl 5):20-28.

32 Rustin GJ, Nelstrop AE, Tuxen MK, et al. Defining progression of ovarian carcinoma during follow-up according to CA 125: a North Thames Ovary Group Study. Ann Oncol 1996;7:361-364.

33 Hoskins WJ. Epithelial ovarian carcinoma: principles of primary surgery. Gynecol Oncol 1994;55:S91-S96.

34 Hoskins WJ, McGuire WP, Brady MF, et al. The effect of diameter of largest residual disease on survival after primary cytoreductive surgery in patients with suboptimal residual epithelial ovarian carcinoma. Am J Obstet Gynecol 1994;170:974-979; discussion 9-80.

35 Rosen DG, Huang X, Deavers MT, et al. Validation of tissue microarray technology in ovarian carcinoma. Mod Pathol 2004;17:790-797.

36 Shimizu Y, Kamoi S, Amada S, et al. Toward the development of a universal grading system for ovarian epithelial carcinoma. I. Prognostic significance of histopathologic features-problems involved in the architectural grading system. Gynecol Oncol 1998;70:2-12.

37 Silva EG, Gershenson DM. Standardized histologic grading of epithelial ovarian cancer: elusive after all these years. Gynecol Oncol 1998;70:1.

38 Kline RC, Wharton JT, Atkinson EN, et al. Endometrioid carcinoma of the ovary: retrospective review of 145 cases. Gynecol Oncol 1990;39:337-346.

39 Podratz KC, Malkasian Jr GD, Hilton JF, et al. Secondlook laparotomy in ovarian cancer: evaluation of pathologic variables. Am J Obstet Gynecol 1985;152: 230-238.

40 Shimizu Y, Kamoi S, Amada S, et al. Toward the development of a universal grading system for ovarian epithelial carcinoma: testing of a proposed system in a series of 461 patients with uniform treatment and follow-up. Cancer 1998;82:893-901.

41 Malkasian Jr GD, Melton 3rd LJ, O’Brien PC, et al. Prognostic significance of histologic classification and grading of epithelial malignancies of the ovary. Am J Obstet Gynecol 1984;149:274-284.

42 Ozols RF, Garvin AJ, Costa J, et al. Advanced ovarian cancer: correlation of histologic grade with response to therapy and survival. Cancer 1980;45:572-581.

43 Mauch PM, Ehrmann RL, Griffiths CT, et al. Radiation therapy in stage II ovarian carcinoma: the influence of histologic grade. Cancer 1980;45:1344-1351.

44 Bichel P, Jakobsen A. A new histologic grading index in ovarian carcinoma. Int J Gynecol Pathol 1989;8: 147-155.

45 Podratz KC, Malkasian GD Jr, Hilton JF, et al. Second-look laparotomy in ovarian cancer: evaluation of pathology variables. Am J Obstet Gynecol 1985;152:230-238.

$46 \mathrm{Fu}$ YS, Stock RJ, Reagan JW, et al. Significance of squamous components in endometrioid carcinoma of the ovary. Cancer 1979;44:614-621.

47 Brachtel EF, Sanchez-Estevez C, Moreno-Bueno G, et al. Distinct molecular alterations in complex endometrial hyperplasia (CEH) with and without immature squamous metaplasia (squamous morules). Am J Surg Pathol 2005;29:1322-1329.

48 Fodde R, Smits R, Clevers H. APC, signal transduction and genetic instability in colorectal cancer. Nat Rev Cancer 2001;1:55-67.

49 Sparks AB, Morin PJ, Vogelstein B, et al. Mutational analysis of the APC/beta-catenin/Tcf pathway in colorectal cancer. Cancer Res 1998;58:1130-1134.

50 Rubinfeld B, Robbins P, El-Gamil M, et al. Stabilization of beta-catenin by genetic defects in melanoma cell lines. Science 1997;275:1790-1792.

51 Zurawel RH, Chiappa SA, Allen C, et al. Sporadic medulloblastomas contain oncogenic beta-catenin mutations. Cancer Res 1998;58:896-899.

52 Fukuchi T, Sakamoto M, Tsuda H, et al. Beta-catenin mutati in carcinoma of the uterine endometrium. Cancer Res 1998;58:3526-3528.

53 Miyoshi Y, Iwao K, Nagasawa Y, et al. Activation of the beta-catenin gene in primary hepatocellular carcinomas by somatic alterations involving exon 3. Cancer Res 1998;58:2524-2527.

54 Faleiro-Rodrigues C, Macedo-Pinto I, Pereira D, et al. Association of E-cadherin and beta-catenin immunoexpression with clinicopathologic features in primary ovarian carcinomas. Hum Pathol 2004;35:663-669.

55 Faleiro-Rodrigues C, Macedo-Pinto I, Pereira D, et al. Loss of beta-catenin is associated with poor survival in ovarian carcinomas. Int J Gynecol Pathol 2004;23:337-346.

56 Marques FR, Fonsechi-Carvasan GA, De Angelo Andrade LA, et al. Immunohistochemical patterns for alpha- and beta-catenin, E- and N-cadherin expression in ovarian epithelial tumors. Gynecol Oncol 2004; 94:16-24.

57 Moreno-Bueno G, Gamallo C, Perez-Gallego L, et al. beta-Catenin expression pattern, beta-catenin gene mutations, and microsatellite instability in endometrioid ovarian carcinomas and synchronous endometrial carcinomas. Diagn Mol Pathol 2001;10:116-122.

58 Emmert-Buck MR, Chuaqui R, Zhuang Z, et al. Molecular analysis of synchronous uterine and ovarian endometrioid tumors. Int J Gynecol Pathol 1997;16:143-148. 